\title{
First Look at the Beam Test Results of the FPIX2 Readout Chip for the BTeV Silicon Pixel Detector
}

\author{
L. Uplegger, J.A. Appel, M. Artuso, G. Cardoso, H.P. Cease, G. Chiodini, D.C. Christian, \\ D.A. Cinabro, R. Coluccia, J. Hoff, S. Kwan, S. Magni, A. Mekkaoui, D. Menasce, \\ C. Newsom, V. Papavassiliou, A. Schreiner, M.A. Turqueti, R. Yarema, J.C. Wang
}

\begin{abstract}
High energy and nuclear physics experiments need tracking devices with excellent spatial precision and readout speed in the face of ever-higher track densities and increased radiation environments. The new generation of hybrid pixel detectors (arrays of silicon diodes bump bonded to arrays of front-end electronic cells) is a technology able to meet these challenges. We report the first results of the $\mathrm{BTeV}$ silicon pixel detector beam test carried out at Fermilab in summer 2004. Tests were performed using a $120 \mathrm{GeV} / \mathrm{c}$ proton beam incident on a 6 planes pixel detector telescope. The last prototype developed for the BTeV experiment (FPIX2) is tested in the middle of the telescope. There is no external trigger and events were built using the time-stamp information provided by the readout chips.
\end{abstract}

\section{INTRODUCTION}

$\mathrm{B}$ $\mathrm{TeV}$ is approved to run at the Fermilab Tevatron collider [1]. One of the key elements of $\mathrm{BTeV}$ is its vertex detector based on hybrid silicon pixel devices. It provides high precision space points for the reconstruction of tracks in close proximity to the interaction region. The vertex detector consists of 30 stations of silicon pixel detectors distributed along the interaction region. Over the last years Fermilab has gone through a series of iterations of prototype pixel readout chips (FPIX0, FPIX1, preFPIX2, FPIX2) [2]. In summer 2004 the latest prototype (FPIX2) was tested in a beam to study the spatial resolution and the functionality close to the real conditions of operation that are envisaged in the experiment. To study the spatial resolution a reference

Manuscript received November 15, 2004. This work was supported in part by the Universities Research Association Inc. under Contract DE-AC0276CH03000 with the United States Department of Energy.

J.A. Appel, G. Cardoso, H.P. Cease, D.C. Christian, J. Hoff, S. Kwan, A. Mekkaoui, M.A. Turqueti, L. Uplegger, R. Yarema are with the Fermi National Acclerator Laboratory, Batavia, IL 60510 USA (e-mail uplegger@fnal.gov).

M. Artuso, J. Wang are with the Syracuse University, Syracuse, NY 13441130, USA.

G. Chiodini, M. Coluccia are with the INFN of Lecce, via Arnesano I, 73100 Lecce, Italy.

D.A. Cinabro, A. Schreiner are with the Wayne State University, Detroit, MI 48202, USA.

S. Magni, D. Menasce are with the INFN of Milano, via Celoria 16, 20133 Milano, Italy.

C. Newsom is with University of Iowa, Iowa City, IA 52242, Usa.

V. Papavassiliou is with the New Mexico State University, Las Cruces, NM 88003-8001, USA. telescope composed of FPIX1 readout chips bonded to pixel sensors was used. A PCI based data acquisition system (DAQ) was developed to record data using the time-stamp information provided by the readout chips present in the system. The timestamp determines the time distribution of the particles in the beam. The DAQ built events using the time-stamp information only and no external trigger was used. Two scintillation counters were also installed to allow time-walk studies. Since the detector will operate at low temperatures a cooling apparatus was installed to study the effect of the temperature of the detector under test.

\section{EXPERIMENTAL SETUP}

The tests were performed in the summer of 2004 in the MTest beam line at Fermilab with $120 \mathrm{GeV} / \mathrm{c}$ protons incident on a 6 planes pixel-detector telescope. This telescope is based on FPIX1 chips, with $50 \mu \mathrm{m} \times 400 \mu \mathrm{m}$ pixel size, arranged in two sections with the detector under test placed between the two stations as shown in Fig. 1.

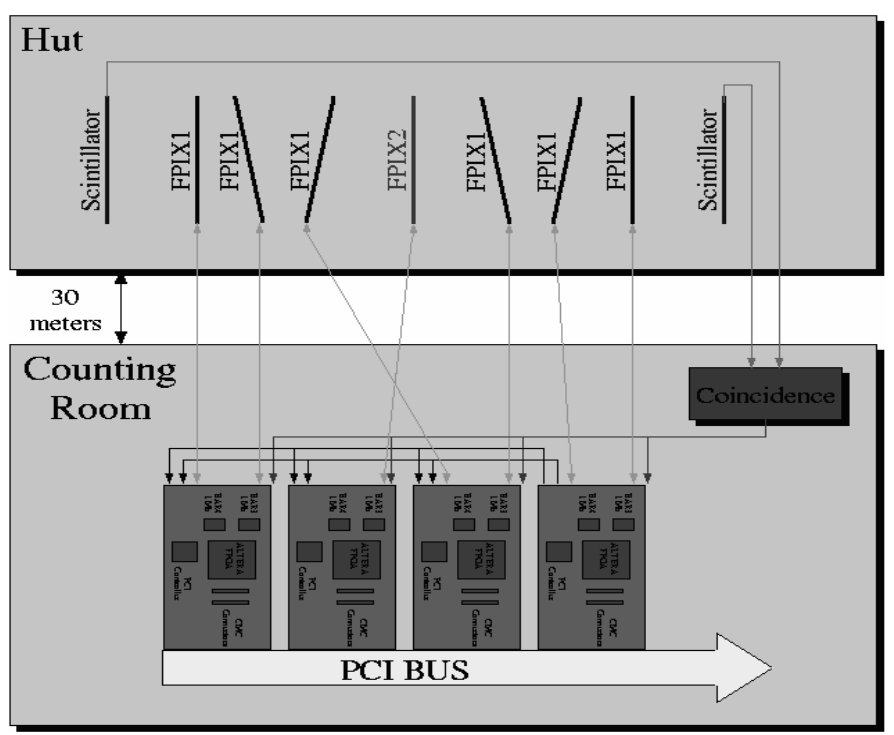

Fig. 1. Schematic drawing of the test-beam data acquisition system. The FPIX2 plane (device under test) is placed between six FPIX1 planes. 
The first and last FPIX1 planes are placed with the $50 \mu \mathrm{m}$ pixel side oriented to measure the vertical (y) coordinate while all the others were oriented to measure the horizontal coordinate (x) with the small pixel side. The measuring $\mathrm{x}$ telescope planes where rotated to 10 degrees to achieve an optimum resolution.

The FPIX1 readout chips were bump bonded to silicon sensors. FPIX1 has 18 columns and 160 rows and its spatial resolution has already been studied in the 1999 beam test [3]. Each FPIX1 cell contains an amplifier and four comparators which form an internal 2-bit flash ADC. The FPIX2 chips were also bump bonded to sensors and have 22 columns and 128 rows. Eight internal comparators form a 3 bit flash ADC. The readout is serialized and can run at a frequency of 70 MHz. During the test beam operations the readout frequency was set to $25 \mathrm{MHz}$. The pixel detectors tested have $50 \mu \mathrm{m} \times$ $400 \mu \mathrm{m}$ pixels and they use the $\mathrm{n}+/ \mathrm{n} / \mathrm{p}^{+}$technology. The sensor thickness is about $245 \mu \mathrm{m} \pm 10 \mu \mathrm{m}$.

The detectors were mounted on printed circuit boards, and placed inside an aluminum dark box (see Fig. 2, Fig. 3, Fig. 4 and Fig. 5).

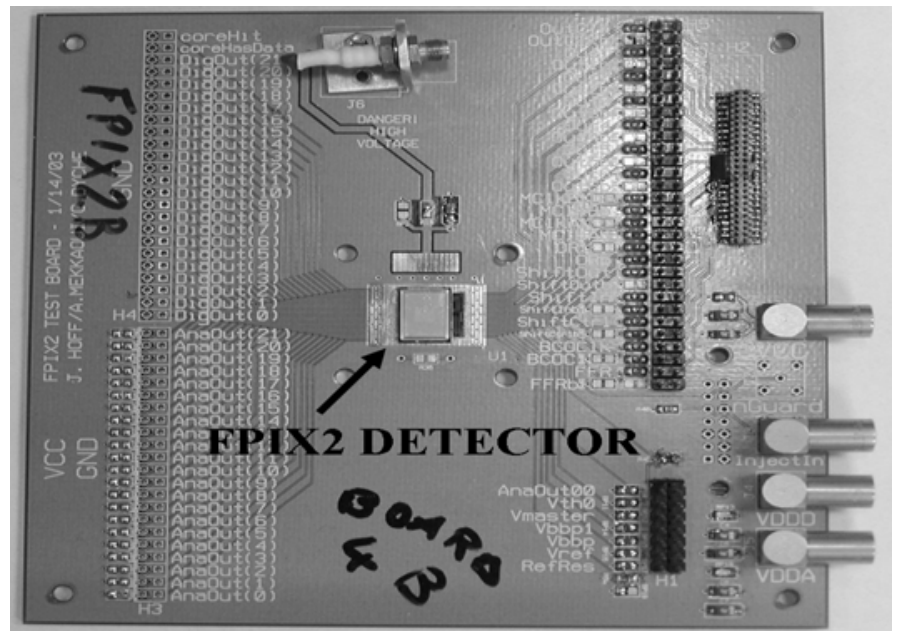

Fig. 2. FPIX2 printed circuit board with the FPIX2 detector in the center.

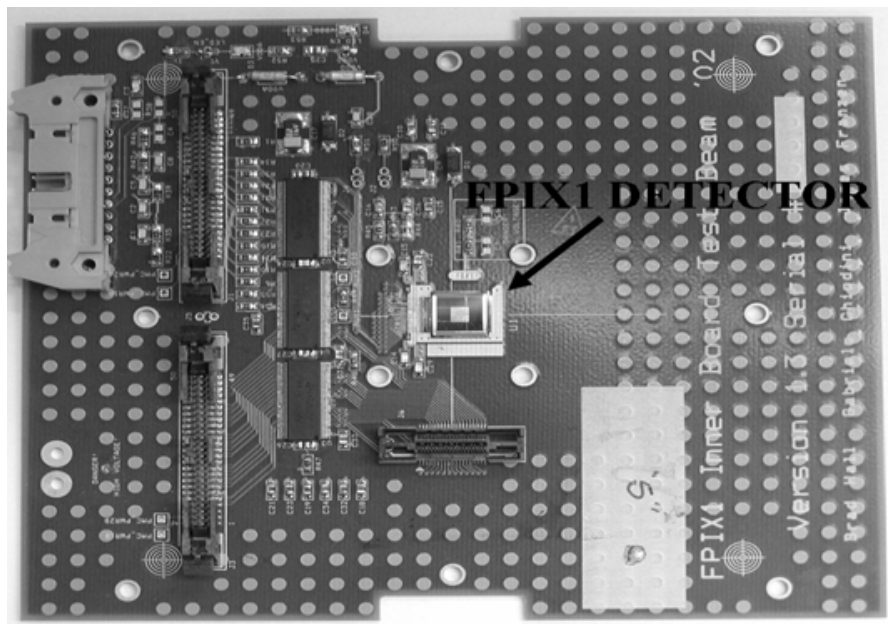

Fig. 3. FPIX1 printed circuit board with the FPIX1 detector in the center.

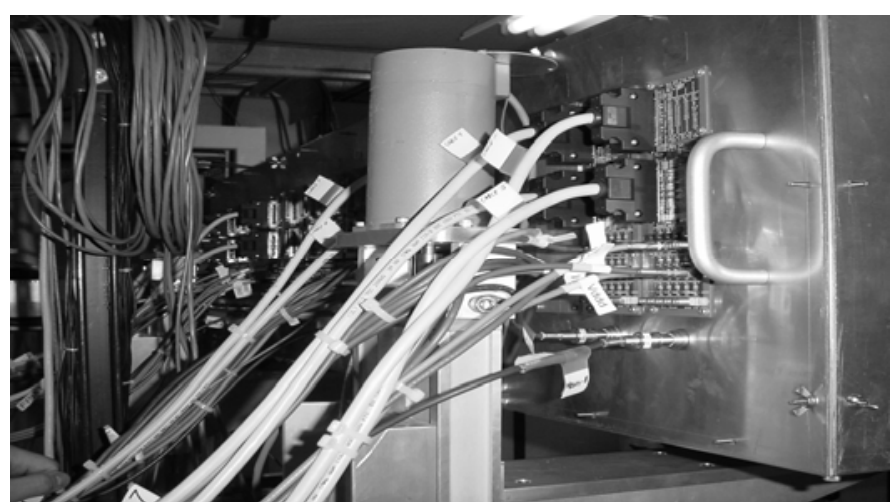

Fig. 4. Telescope support structure-

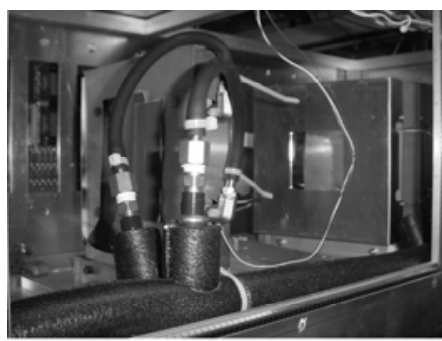

(A)

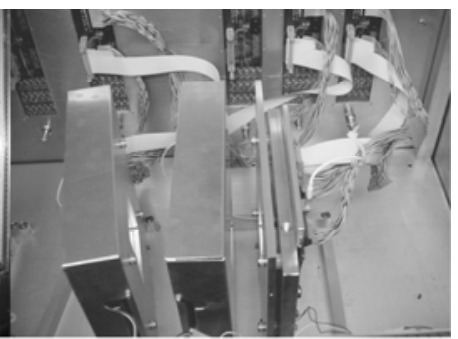

(B)
Fig. 5. View of the inside of the support structure. A) Cooling system. B) Three detectors in their respective boxes are shown.

The pixel planes inside the box were connected through 30 meter cables to PCI cards located in the counting room (see Fig. 1).

A special characteristic of this system is the PCI based DAQ system developed at Fermilab. The field programmable gate array (FPGA) chips on the PCI boards provide flexibility in using and operating the DAQ system. The DAQ system is based on a combination of two cards: the PCI test adapter (PTA) and the programmable mezzanine card (PMC) (see Fig. $6)$.

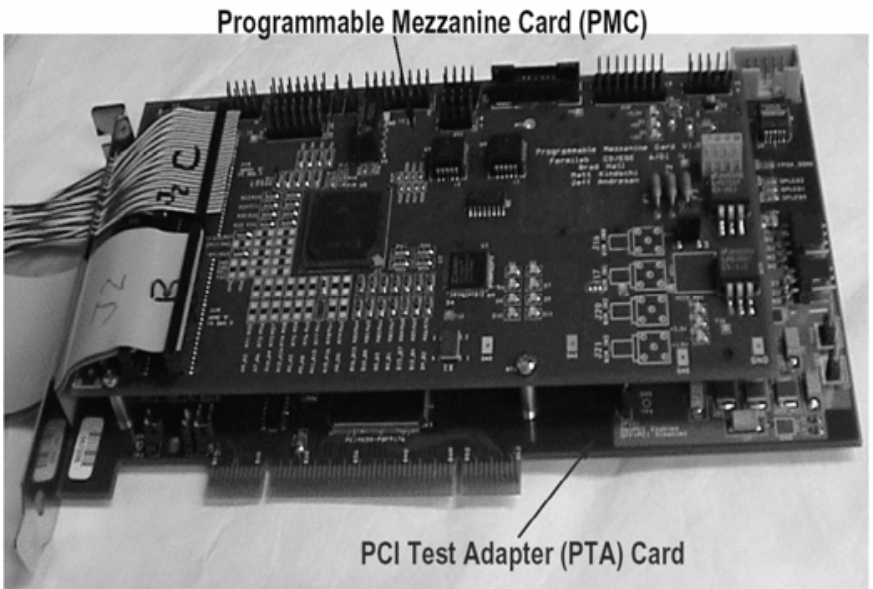

Fig. 6. PMC card mated on a PCI card.

The PTA is responsible for interfacing with the PCI bus and for temporarily storing the data from the pixel chips. The PMC complements the functionalities of the PTA by implementing 
the communication layer protocol to interface with the FPIX1 and FPIX2 front-end electronics.

Every PTA-PMC set can control up to two FPIX1 detectors and up to four FPIX2 detectors.

The general scheme of the DAQ has been implemented having in mind a continuous data flow produced by the pixel planes. Hence, the goal is to operate the system as close as possible to the real operation conditions of the $\mathrm{BTeV}$ experiment, where data is collected and used for the eventselection trigger as soon as it is available. The PCI based DAQ system builds events in the absence of any external trigger that indicates the presence of a particle passing through the pixel system. In order to build an event, the readout chips provide a time-stamp which marks the time when a pixel cell is above threshold. A time-stamp is an internal counter in the readout chip that is incremented with a clock provided by a master PMC. This master PMC distributes the same clock to all other PMCs, which then distributes it synchronously to all the detectors.

The DAQ software [4] runs on a Linux PC, it is implemented in $\mathrm{C}^{++}$and it is designed around three main processes: Producer, Consumer, and Logger. The Producer controls the PTA cards installed. It sends commands to the FPGAs and receives data from the pixel planes. Data are then stored in a shared memory. The Consumer process reads and analyzes the data, building events that are then stored on disk. The Logger receives messages from the other processes and notifies the user of any error in the system. Fig. 7 shows the Data Acquisition Graphical Interface developed to manage the different processes.

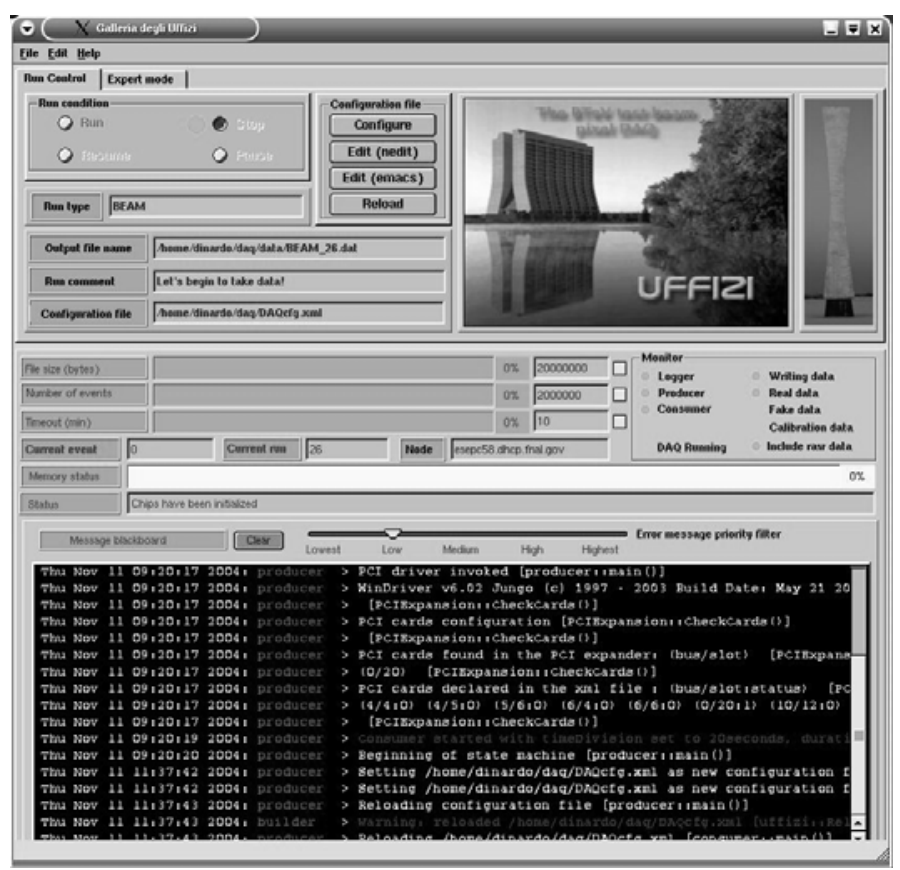

Fig. 7. DAQ graphical user interface.

\section{DATA ANALYSIS AND FIRST RESUlTS}

\section{A. General Description}

The track position at each plane in the telescope is reconstructed using linear charge weighting. Each track is fitted to a straight line using the Kalman-filter technique and events with a good track- $\chi^{2}$ are retained for further analysis. The Kalman filter includes information from the pixel telescope without considering the device under test. The track parameters allow the projection of the track position $\left(\mathrm{x}_{\mathrm{T}}, \mathrm{y}_{\mathrm{T}}\right)$ at which the beam intersects the sensor being characterized. Although the pixel detectors measure two coordinates, the following discussion will focus on the resolution measured in the direction with smaller pitch - which was oriented to measure the horizontal $(\mathrm{x})$ coordinate.

The measured spatial resolution of a pixel plane is the difference between the reconstructed pixel position $\mathrm{x}_{\mathrm{p}}$, and the track position $\mathrm{x}_{\mathrm{T}}$, known with an accuracy of about $4 \mu \mathrm{m}$, limited by multiple Coulomb scattering from the material in the telescope and the intrinsic hit resolution of the various tracking stations. This prediction uncertainty is not subtracted in the results shown below.

\section{B. Pixel Occupancy}

In this section we show the relation of the number of pixels in a cluster in the same column as a function of the track incident angle. The charge deposited by a single track is often shared by more than one pixel. The amount of charge sharing is determined by the number of pixel cells crossed by the track, and by the diffusion induced spread of the electrons drifting in the silicon. Fig. 8 shows the number of rows in a cluster as a function of the incident track angle, measured for the FPIX2 detector under test (DUT).

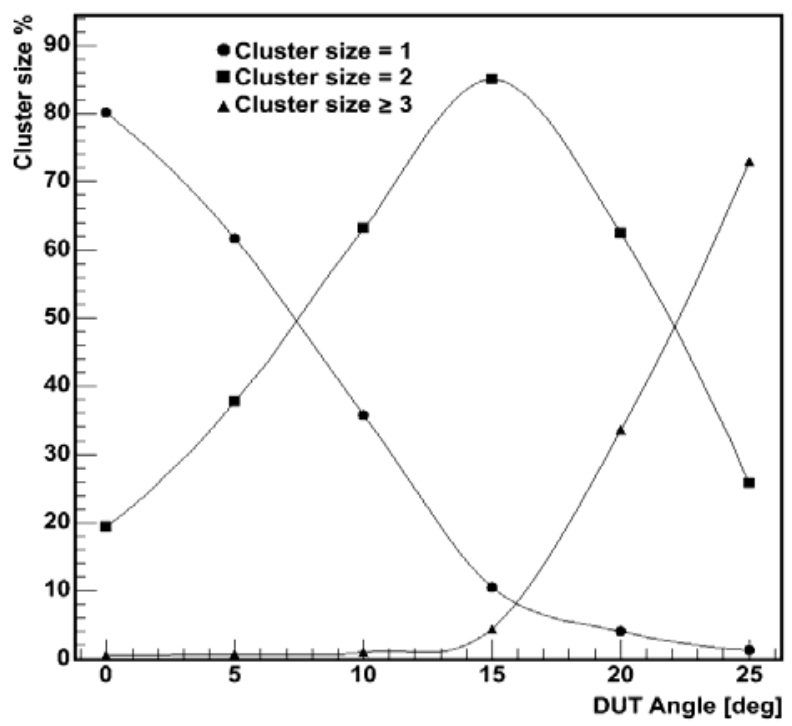

Fig. 8. Cluster row multiplicity percentage for various angles of the FPIX2 detector under test $\left(\mathrm{V}_{\text {bias }}=250 \mathrm{~V}\right.$ and $\left.\mathrm{Q}_{\mathrm{thr}}=1800 \mathrm{e}^{-}\right)$. 
T: $23^{\circ} \mathrm{C}$ BIAS: $300 \mathrm{~V}$ ANGLE: $0^{\circ}$

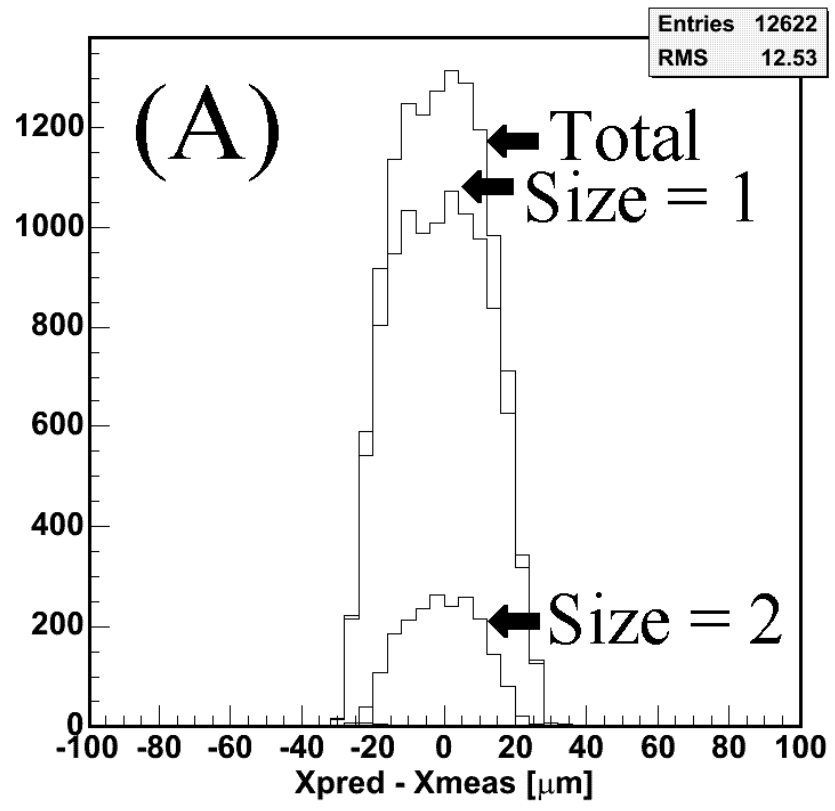

T: $23^{\circ} \mathrm{C}$ BIAS: $300 \mathrm{~V}$ ANGLE: $15^{\circ}$

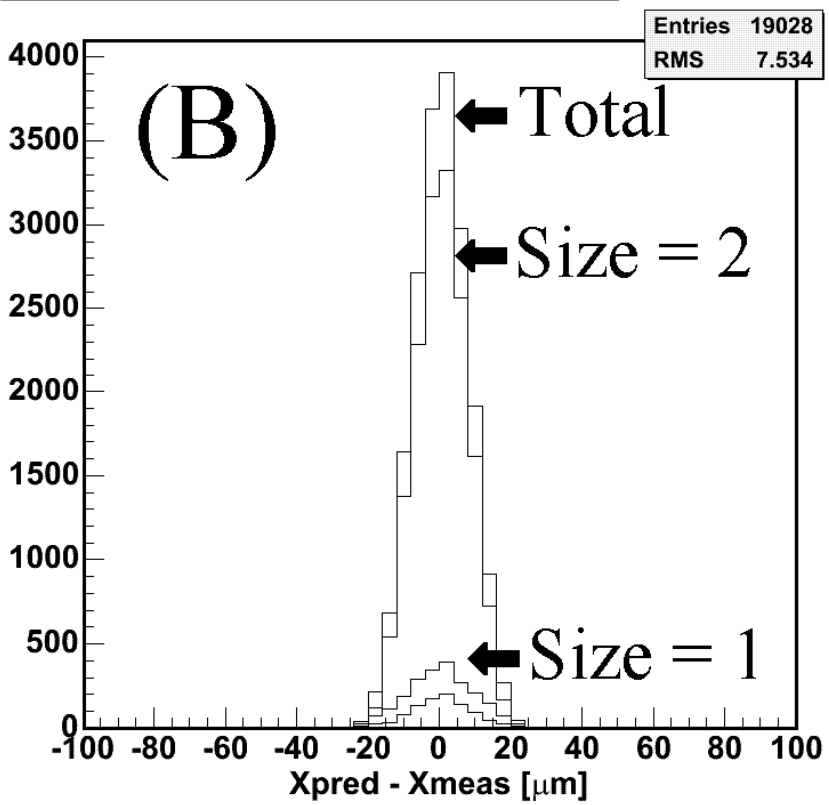

Fig. 9. Cluster size distributions at two different angles. - A) The total distribution is dominated at $0^{\circ}$ by the clusters with size $=1$ and the RMS resolution is

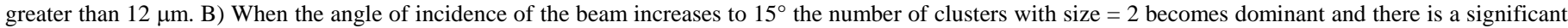
improvement in the RMS resolution $=7.5 \mu \mathrm{m}$.

Fig. 9 shows the improvement in RMS resolution when the dominant clusters changes from width $=1$ to width $=2$.

As shown in Fig. 9 the best resolution is achieved when a particle interacting with the detector deposits the charge in more than one pixel cell.

\section{Position Resolution at Different Temperatures and Bias Applied to the Sensor.}

We also studied the spatial resolution achieved with an FPIX2 pixel detector for tracks incident at 0, 5, 10, 15, 20 and 25 degrees relative to the normal to the pixel sensor and for different temperatures and bias voltages applied to the sensor. More than 12,000 tracks were used for each measurement. Only tracks that hit all 7 planes (6 in the telescope plus the device under test) were used in this analysis. The resolutions plotted in Fig. 10 and Fig. 11 are the RMS values of the total distributions obtained from the histograms of the residuals. The results shown in Fig. 10 for the FPIX2 detector were taken at three different temperatures: room temperature $\left(23^{\circ} \mathrm{C}\right), 5^{\circ} \mathrm{C}$ and $-5^{\circ} \mathrm{C}$. The bias applied to the sensor was $250 \mathrm{~V}$, and the threshold was set to $\sim 1800 \mathrm{e}^{-}$.

Fig. 10 shows that the effect of temperature is negligible compared to the effect caused by the incident angle of the beam. The resolution obtained, without the telescope subtraction, varies from $12 \mu \mathrm{m}$ to $7.5 \mu \mathrm{m}$.

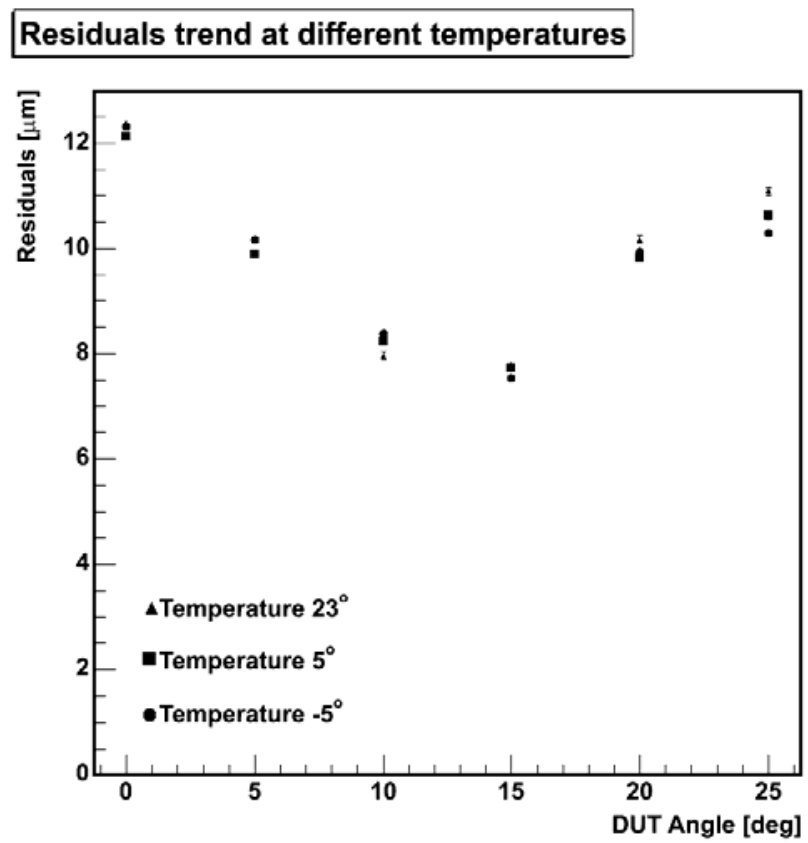

Fig. 10. Detector resolution at various angles for different temperatures applied to the FPIX2 detector.

A similar study has been performed for different bias voltages applied to the sensor. The depletion voltage for the sensors bump bonded to the FPIX2 is around $150 \mathrm{~V}$ and the measurements were taken at $200 \mathrm{~V}, 250 \mathrm{~V}$, and $300 \mathrm{~V}$. The detector was at room temperature and the threshold was set to $\sim 1800 \mathrm{e}^{-}$. Fig. 11 shows the results obtained for different biases applied at various angles. 


\section{Residuals trend at different sensor biases}

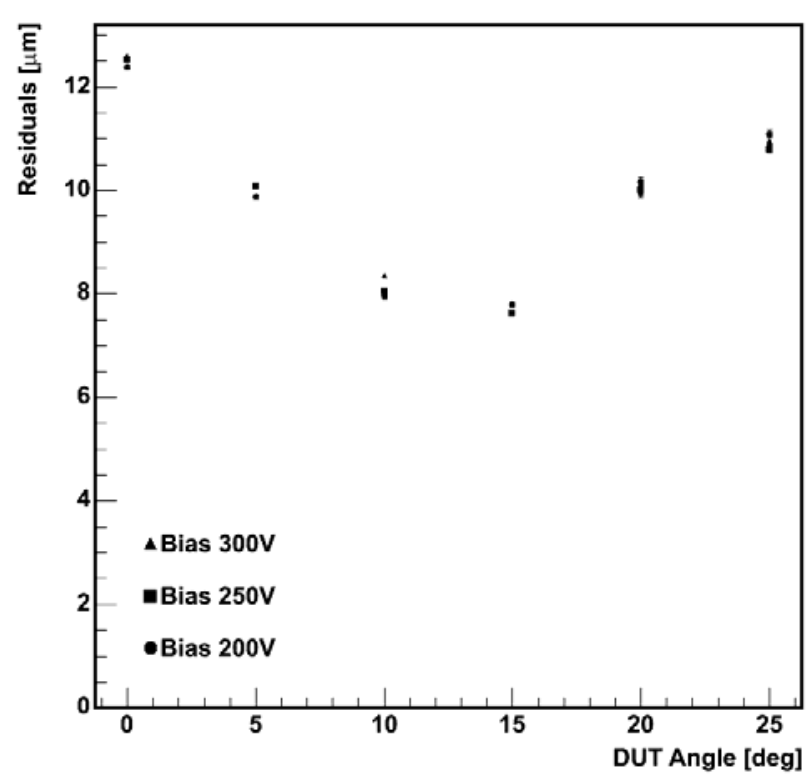

Fig. 11. Detector resolution at various angles for different applied biases.

Also in the case of applied bias, the main effect on the resolution is caused by the incident angle of the beam particle, while the bias applied has a small effect.

\section{CONCLUSIONS}

We have obtained the first results from the summer 2004 beam test of a $\mathrm{BTeV}$ hybrid silicon pixel detector at Fermilab. We were able to build a beam-tracking telescope with pixel detectors only, and test the latest version of the $\mathrm{BTeV}$ readout chip, FPIX2. The results presented in this paper show that the main effect on the spatial resolution is caused by the angle of incidence of the beam particle. The effect of changing the temperature from $-5^{\circ} \mathrm{C}$ to $23^{\circ} \mathrm{C}$ on the track resolution is negligible. Furthermore, we also studied the effect of the bias voltage applied to the sensor on the track resolution. Similarly, no major effects have been observed for biases above full depletion.

\section{ACKNOWLEDGMENT}

We would like to thank Fermilab for providing us with the dedicated beam time for our test and the excellent infrastructure support.

We thank the controls people, Rich Schmitt, Mark Knapp, Dan Markley, for the support they provided us during the data taking.

We also thank David Slimmer, Terry Tope, Erik Ramberg, Charles Brown and Greg Sellberg.

\section{REFERENCES}

http://www-btev.fnal.gov/public/hep/general/proposal/index.shtml.

[2] D.C. Christian, J.A. Appel, G. Cancelo, S. Kwan, J. Hoff, A. Mekkaoui, et al., "Development of a pixel readout chip for BTeV", Nucl. Instrum. Meth. A435: 144-152, 1999.

[3] G. Chiodini, J.A. Appel, M. Artuso, J.N. Butler, G. Cardoso, H. Cheung, et al., "Beam test results of the BTeV silicon pixel detector", Nucl. Instrum. Meth. A465: 125-130, 2000.

[4] S. Magni, G. Alimonti, G. Chiodini, B.K. Hall, D. Menasce, L. Uplegger, et al., "Pomone, a PCI based data acquisition system", in Proc. IEEE Nuclear Science Symposium, 2003. 